\title{
Reshaping the outskirts of planetary systems
}

\begin{abstract}
C. de la Fuente Marcos and R. de la Fuente Marcos
Universidad Complutense de Madrid, Ciudad Universitaria, 28040 Madrid, Spain

Received 31 October 2000 / Accepted 12 March 2001

Abstract. We present $N$-body calculations of the orbital evolution of a test population of primordial EdgeworthKuiper Belt objects (EKBOs) in a star cluster submitted to the gravitational perturbations of other cluster members, both single stars and binaries. In order to provide initial conditions for the EKBO test population, three original orbital distributions (eccentricity and semi-major axis) are considered. The first resembles a conventional model of the evolution of the solar nebula with eccentricity, $e=0.01$ and semi-major axis uniformly distributed in the range 35-60 AU. The second distribution considers the stage of planet formation after Neptune's outward migration with concentration of solid particles at $39 \mathrm{AU}$ and $e \sim 0.1-0.3$. The third distribution is taken from a non-collisional, two-dimensional simulation of a gaseous protoplanetary disk in which solid particles beyond $60 \mathrm{AU}$ are perturbed by the growing proto-planets, Uranus and Neptune. Metric particles are trapped in an outer resonance with radial distance of about 48.5 AU and eccentricity of 0.16. Stellar encounters lead to significant modification of the primordial orbital distribution only if the Sun was born in a typical open cluster but not in an association. Our results suggest that the observed orbital distribution of the scattered EKBOs may be the result of these multi-body gravitational interactions in the Sun's parent star cluster. On the other hand, the circular shape of the Edgeworth-Kuiper Belt-like structure found around $\epsilon$ Eridani indicates that this star was born in a small cluster or association.
\end{abstract}

Key words. minor planets, asteroids - comets: general - Kuiper belt, Oort cloud - planets and satellites: general - solar system: general - open clusters and associations: general

\section{Introduction}

Stars are born in a clustered environment. In the Galactic disk, open clusters, which are loose, irregular clusters that typically contain from a few tens to several thousands of stars, are being formed and disintegrated continuously. The majority of galactic open clusters have ages younger than 0.8 Gyr (Mermilliod 2000); neglecting observational selection effects, it suggests that most of the stars are born in clusters with initial population $\leq 1000$ stars (de la Fuente Marcos 1997). On the other hand, it is thought that planets arise before the host protostar leaves its parent star cluster (after a few Myr). Standard theories of planet formation require a disk of gas and dust to form planets by coagulation of small particles due to gas drag and perturbations of the particle orbits (Weidenschilling 1980). Out of these particles, kilometre-sized planetesimals (asteroid-like objects) are formed. After planet formation the remnant planetesimals can generate asteroidal belts (e.g. the low inclination population of trans-Neptunian objects, the Edgeworth-Kuiper

Send offprint requests to: C. de la Fuente Marcos, e-mail: nbplanet@eucmos.sim.ucm.es
Belt). The orbits of these primordial particles are almost circular due to viscous dissipation; this fact suggests that newly formed planets should also have small eccentricities. However, orbits of remnant planetesimals evolve in a (very likely) multi-planet environment so they are expected to be modified as a result of gravitational resonances. On the other hand, the dynamics of the parent star clusters is dominated by distant and close encounters among the cluster members. These multi-body gravitational interactions can modify the original distribution of orbital elements for both primordial binaries and planetary systems in a very efficient way (de la Fuente Marcos \& de la Fuente Marcos 2000a, 2001). The orbital modification process is more important for wider systems therefore some dynamical signatures (reshaping) on the outer regions of planetary systems (Edgeworth-Kuiper Belt-like structures) are expected.

In the last few years, a large number of transNeptunian and scattered disk objects have been discovered (see Jewitt \& Luu 2000 for a recent review). These observational results suggest that the outer solar system is richly populated by small bodies. They are believed to be icy planetesimals remaining from the time preceding 
planet formation. These bodies are in wide orbits, mainly around $40 \mathrm{AU}$, therefore their orbits may be perturbed very efficiently by other stars in clusters. In this paper, we use numerical simulations to show that the observational orbital distribution of the population of the Scattered Edgeworth-Kuiper Belt may be the result of stellar encounters of the proto-Sun with other stars in its parent star cluster. In addition, we study the general problem of reshaping the outer regions of planetary systems as a result of dynamical interactions in star clusters.

In the following section, details of the numerical calculations performed are given. Section 3 presents different models of planet formation. In Sect. 4 we analyze the results of our calculations. In a concluding section a discussion of the implications of these results is presented.

\section{Modeling}

The orbital modification of a primordial distribution of Edgeworth-Kuiper Belt objects (EKBOs) could in principle be attacked as a statistical process by performing a large number of separate planetary scattering (2 or 3body stellar encounters) experiments using a Monte-Carlo scheme to select the input parameters. This approach has been recently taken by Ida et al. (2000a) and Adams \& Laughlin (2001).

Ida et al. (2000a) consider interactions of a star (the proto-Sun) having a planetesimal disk that encounters a passing single star (2-body stellar encounter). They have performed a series of numerical simulations to test the effect of stellar companion encounters in protoplanetary disks. In this work, they considered collisionless particles (corresponding to planetesimals), orbiting initially on coplanar circles around a primary star (the proto-Sun). The particle disk encounters a star and the orbital changes of the test particles are integrated taking into account the gravitational forces of the primary and the companion star by using a fourth-order predictor corrector scheme. In general, however, this experimental approach implies to state that 2-body stellar encounters are the only ones to contribute to Edgeworth-Kuiper Belt reshaping. This starting assumption is highly arguable according to previous calculations by the authors in the related topic of orbital modification in Jovian-like systems (de la Fuente Marcos \& de la Fuente Marcos 1997, 1998a, 2000a, 2001) because the formation of temporarily stable hierarchical configurations (like the ones required for significant orbital modification) requires, at least, 5-bodies.

On the other hand, Adams \& Laughlin (2001) using a planet scattering (3-body stellar encounters) code (Laughlin \& Adams 1998, 2000) have calculated the cross sections for the scattering of EKBOs on initially circular orbits with radii in the range $30 \mathrm{AU} \leq a \leq 70$ AU by gravitational interaction with passing binary stars in the birth cluster. Their experimental approach implies to state that 3-body stellar encounters are the only ones to contribute to EKBO orbit modification.
Although the Monte-Carlo approach provides useful information about the outcome of these gravitational interactions, the orbital modification problem is best treated through the direct numerical integration of entire open clusters. In this way, we do not make any assumptions about the type of dynamical interactions involved.

In this paper and instead of calculating the evolution of several cluster models with different populations and sizes we consider a sample of models calculated with NBODY5 (Aarseth 1994) only differing in the value of the seed number for generating initial conditions. For our study we choose an open cluster model with $N=800$ objects (10\% single stars, $40 \%$ binaries, $50 \%$ EKB test systems). For simplicity, the systems studied in this research consists of one test EKBO and its host star. A specified mass range of 0.08-15.0 $M_{\odot}$ is adopted for the stars and their masses are sampled from a realistic mass function (de la Fuente Marcos \& de la Fuente Marcos 2000b). To generate both initial positions and velocities we assume a Plummer model (Aarseth et al. 1974), with the ratio of the total kinetic and potential energy fixed to 0.25 . The halfmass radius is $R_{\mathrm{h}} \sim 0.8 \mathrm{pc}$, and the typical rms velocity at half-mass radius, $v$, is $\sim 1.1 \mathrm{~km} \mathrm{~s}^{-1}$, with a corresponding crossing time $\left(2 R_{\mathrm{h}} / v\right)$ of about $1.410^{6}$ yr. We select $40 \%$ primordial binaries as a plausible binary percentage for open clusters, with semi-major axis $a_{b}$ in the range 170$1030 \mathrm{AU}$. This compares with $a_{\mathrm{h}}=2 R_{\mathrm{h}} / N \sim 413 \mathrm{AU}$ for a typical hard binary ( $60 \%$ hard binaries). The cluster is assumed to be in a circular orbit in the solar neighbourhood, with a standard linearized tidal force added to the equations of motion which are expressed in rotating coordinates (Aarseth 1994). Escaping objects (binaries, EKB test systems, single stars and lone asteroids) outside twice the classical tidal radius ( $\sim 7 \mathrm{pc})$ are removed from the calculation in order to focus attention on the bound cluster. Stellar evolution is included by means of the fast lookup tables developed by Eggleton et al. (1989). This fitting scheme yields the stellar radius, luminosity, mass and classification type as a function of the initial mass and age. As pointed out before, the EKB systems studied in this paper consist of only one test object of about $410^{20} \mathrm{~kg}$ (assuming a density of $2000 \mathrm{~kg} \mathrm{~m}^{-3}$ it corresponds to a radius of about $300 \mathrm{~km}$ ) and its host star. In order to select appropriate initial conditions for the test particles we discuss different models of planet formation in the following section.

\section{Planet formation: different scenarios}

In the frame of one of the conventional models, planetesimal formation is thought to be the result of a gravitational instability in a dense dust layer, leading to fragmentation into kilometre-sized bodies (Safronov 1969; Goldreich \& Ward 1973). In this scenario, when proto-Neptune was already formed the planetesimals have grown to comparable sizes of the present EKBOs. This model suggests that primordial EKBOs were distributed (but not uniformly) in 
the outer disk with low eccentricities $(\leq 0.01)$ as a result of gas drag.

On the other hand, the formation of planetesimals can also be explained by collisional aggregation of particles. In fact, Weidenschilling (1980) was the first to note the difficulty with the particle layer instability. Cuzzi et al. (1993) and Weidenschilling (1995) demonstrated that such an instability is unlikely to occur. Particles must grow to at least metre size before they can decouple from shearinduced turbulence and slow to the low velocities required for the instability to occur. The radial velocity dispersion induced by drag will further delay the onset of instability until the mean size is in the range of $10-100 \mathrm{~m}$. In the collisional scenario (see e.g. Beckwith et al. 2000) particles are built up by coagulation and sticking in the early stages. In about $10^{4} \mathrm{yr}$, particles can grow from micron size to metre size through a variety of collisional processes: Brownian motion, settling, turbulence and radial migration. The dominant mode depends on physical conditions within the disk. After another $10^{4} \mathrm{yr}$ or so, the small bodies can attain sizes of order of kilometres. Beyond $1 \mathrm{~km}$, gravitational attraction causes the planetesimals to grow by pairwise collisions and later by runaway growth until some planets will be present after $1 \mathrm{Myr}$.

Recent gas giant planet formation studies have suggested that most of the action in planet formation occurs over 1-16 Myr with formation of planets similar to Jupiter in $t \leq 10 \mathrm{Myr}$. For the conditions of the primitive solar nebula, grain growth from sizes typical of grains in the interstellar medium to metre-size (and larger) occurs in less than $10^{5} \mathrm{yr}$ at distances of $30 \mathrm{AU}$ from the Sun, and it is faster at shorter distances (Weidenschilling 1997; Schmitt et al. 1997). As regards EKBOs, the final output from this collisional scenario is likely similar to that of the instability model. Simplified models of the accretion in the trans-Neptunian region (Stern \& Colwell 1997) suggest that the time scale for the growth of Neptune and other larger bodies in Neptune's region in the ancient solar system may well have been of order several hundred Myr. This model also indicates that the Neptune's growth was not very rapid. On the other hand, recent models by Bryden et al. (2000) suggest that the formation time scale of proto-Neptune would be $\sim 10^{6-7} \mathrm{yr}$.

However, observational results (Trilling et al. 2000) suggest that the actual sequence in planetesimal growth may be more complex. The most extreme example is the system around $\rho^{1}$ Cancri. This 5 Gyr old system has both circumstellar dust and at least one planet. ISO observations detected the disk at $60-90 \mu \mathrm{m}$, modeled as due to $\approx 410^{-5} M_{\oplus}$ of $10-\mu \mathrm{m}$ dust grains lying between 35 and $60 \mathrm{AU}$ from the star with a definite central cavity (Dominik et al. 1998). Another example is the 0.51.0 Gyr old $\epsilon$ Eridani (Greaves et al. 1998) disk. In this system planet formation is ongoing or complete, but some dust $\left(0.01-0.4 M_{\oplus}\right)$ is still present at all radii out to about 115 AU. In addition UX Ori-type stars (spectral types A0-A5) are known to posses significant amounts of metric bodies although they are a few Myr old (Grinin et al. 1996). Comets may form in only a few $10^{5} \mathrm{yr}$ (Weidenschilling 1997), but the time to accrete and/or disperse all the dust is likely to be $>>10^{8} \mathrm{yr}$, which is the period estimated to form large EKBOs (Kenyon \& Luu 1998).

Based on the above observational results, it is not easy to understand how disk grains can have typical millimetre to metre sizes over a long period of time. Therefore, there is a strong observational evidence for circumstellar disks around young stellar objects but, presently, no clear understanding of their evolution. Such disks are thought to be turbulent during a span of their lives (e.g. Lin 1981; Morfill 1985; Boss 1988) as to explain the mass and angular momentum evolution, and also the constraints deduced from the meteoritic data (Palme \& Boynton 1993). Large scale coherent structures have been suggested to grow in these turbulent disks (Barge \& Sommeria 1995) by analogy with what happens in 2D fluid dynamics in which organized structures are known to emerge from random turbulence in rotating shear flows. Striking examples are the persistent atmospheric vortices in the giant planets like Jupiter's Great Red Spot (Ingersoll 1990). This phenomenon can be reproduced in laboratory experiments (Sommeria et al. 1988; Nezlin \& Snezhkin 1993) and explained in terms of statistical mechanics of two dimensional turbulence (Sommeria et al. 1991; Miller et al. 1992).

More recently, the growth of such structures in a Keplerian flow was also simulated numerically when starting from an initially random vorticity field in the incompressible case (Bracco et al. 1998) as well as in the shallow water approximation (Bracco et al. 1999). Barge \& Sommeria (1995) found that large scale anticyclonic vortices can capture and concentrate very efficiently the solid particles of the Nebula and claimed that they could play a central role in the first stages of planetary formation. They conclude that persistent giant vortices could be the most appropriate places for the formation of the planetesimals and the giant-planet cores and, consequently, that the capture-in-vortex mechanism could help explaining the birth of the Giant gas planets in less than a million years. The importance of vortices in planetary formation is now being investigated by other authors (Tanga et al. 1996; Bracco et al. 1998, 1999; Chavanis 2000; Godon \& Livio 1999a, b, 2000; de la Fuente Marcos \& Barge 2001).

Following the standard acceptance, the flow of gas in the Nebula is axisymmetric and the solid particles are drifting inward to the Sun. This drift arises from the systematic difference between the velocity of the gas, which is pressure supported, and that of the solid component, which is not. The resulting lifetime against loss into the Sun is very short for particles whose size ranges from centimetre to metre. For example, in the case of a standard solar nebula, it is less than $10^{3}$ yr for a particle size of the order of $60 \mathrm{~cm}$ and initial radial distance of the order of 5 AU. As pointed out by Cuzzi et al. (1993), the back reaction of the dense sub-layer of the particles onto the gas tends to lengthen the lifetimes, but this effect is weak 
in the size range of interest (and likely weaker for more massive nebulae). In fact this time scale problem seriously questions the continuous growth model, in which the instability of the dust layer does not arise but the planetesimals form in a gradual accumulation of dust and rocks. Indeed, for this scenario to be effective, metre-sized particles have to survive in the Nebula long enough so as to keep growing and reach the size of long-lived bodies. An important effect of this capture-in-vortex confining mechanism is that it avoids metre-sized bodies to fall into the Sun, as occurs during their growth in an axisymmetric nebula. Indeed, giant vortices capture or confine these particles allowing them to grow more easily and to bridge the gap in the metre size range. This planet formation mechanism may be, in principle, the only one able to permit coexistence of planetary bodies and primordial metric (or submetric) solid material. If the four giant planets were formed inside vortices, it is consistent to consider the dynamical effects of growing proto-giant planets (Uranus and Neptune) on metric-size solid material. We may say that the initial conditions arisen from this model are non-conventional but plausible in the frame of the capture-in-vortex model. In order to obtain these initial conditions for a primordial EKBO population we perform a numerical simulation to test the effect of Uranus and Neptune on the solid material in a gaseous protoplanetary disk. The details of these additional calculations are discussed below.

\section{Results}

\subsection{Proto-EKBOs evolution}

If we assume that giant planets were formed inside vortices, metre-sized solid material can coexist with growing giant proto-planets. In this scenario and in order to provide suitable initial conditions for our main $N$-body simulation with NBODY5 we use the two-dimensional, non-collisional code described in de la Fuente Marcos \& de la Fuente Marcos (1998b). This code uses a BulirschStoer scheme (Bulirsch \& Stoer 1966) to solve the motion equations of solid particles submitted to viscous forces and the gravitational interaction of the Sun, Uranus and Neptune. This calculation provides us the location of the primeval, non gravitational, outer resonances beyond Neptune. In these outer resonances where a particle revolves slower than the planets gain and loss of orbital momentum as well as of orbital energy may be kept in equilibrium (Scholl et al. 1993). The time scale for this equilibrium state depends on the values for the planetary masses, for the eccentricity of the planetary orbits, for the particles' radii and depends on the order of the resonance. In order to study whether or not resonance trapping of particles is a plausible mechanism to confine solid material able to form primordial EKBOs we consider the epoch when the masses of proto-Uranus and protoNeptune reach the present values by accreting the gases of the solar nebula. During this stage, drag-induced orbital decay of material from the outer solar nebula pro-

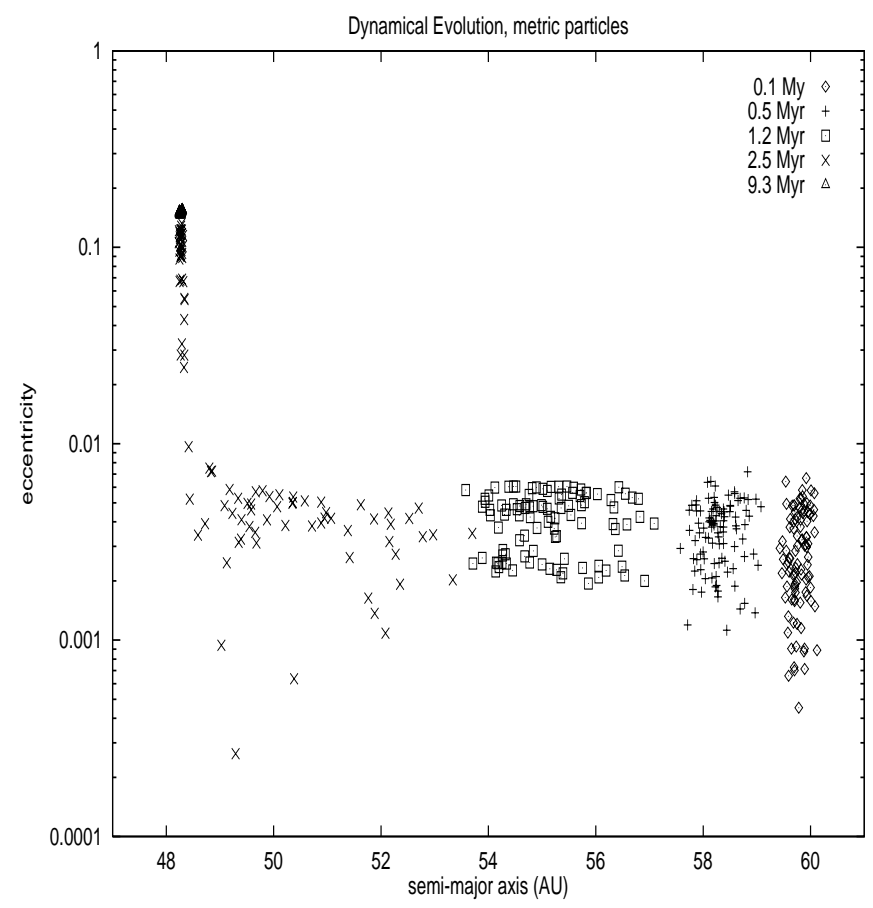

Fig. 1. Orbital evolution of a selected model of proto-EKBOs. Particles are finally trapped in a stable configuration at a mean heliocentric distance of $48.5 \mathrm{AU}$ with an eccentricity of 0.16

vides continuous replenishment of solid particles. We have carried out a series of numerical integrations of coplanar orbits of solid metric particles to investigate the response of the particles, in a protoplanetary disk with radius $R_{\mathrm{D}}=$ $100 \mathrm{AU}$ and mass $M_{\mathrm{D}}=0.001 M_{\odot}$. If giant gaseous, protoplanets are still accreting gas, then a significant fraction of $M_{\mathrm{D}}$ is in gas form.

We have generated a dust particle population orbiting the Sun with initial eccentricity 0.010 and initial heliocentric distance 60 AU submitted to the gravitational attraction of both proto-Uranus and proto-Neptune with masses $10 \%$ of their current value and eccentricities $0.0472,0.0086$, respectively. In our calculations, the initially circular orbits of the test particles are perturbed by the Sun, proto-Uranus and proto-Neptune but do not themselves exert any gravitational forces. Instead of considering a continuous growth model for the giant planets we restrict our calculations to a static combination for the masses of the growing protoplanets. Our qualitative results do not strongly depend on the choice of protoplanetary masses but the quantitative results (order of resonances) depend slightly on these values. We decide to focus our attention in metre-sized particles following Weidenschilling (1994). In his work he argues that cometary objects (likely the ones in the EKB) could form by accretion of primary nuclei or building blocks, tens of metres in size. This theory is consistent with the observed sizes and structure of comet nuclei as well as outbursts and splitting events. Our calculations show that after 7-9 Myr particles are trapped in a stable configuration at a mean heliocentric distance of $48.5 \mathrm{AU}$ with eccentricities in the 


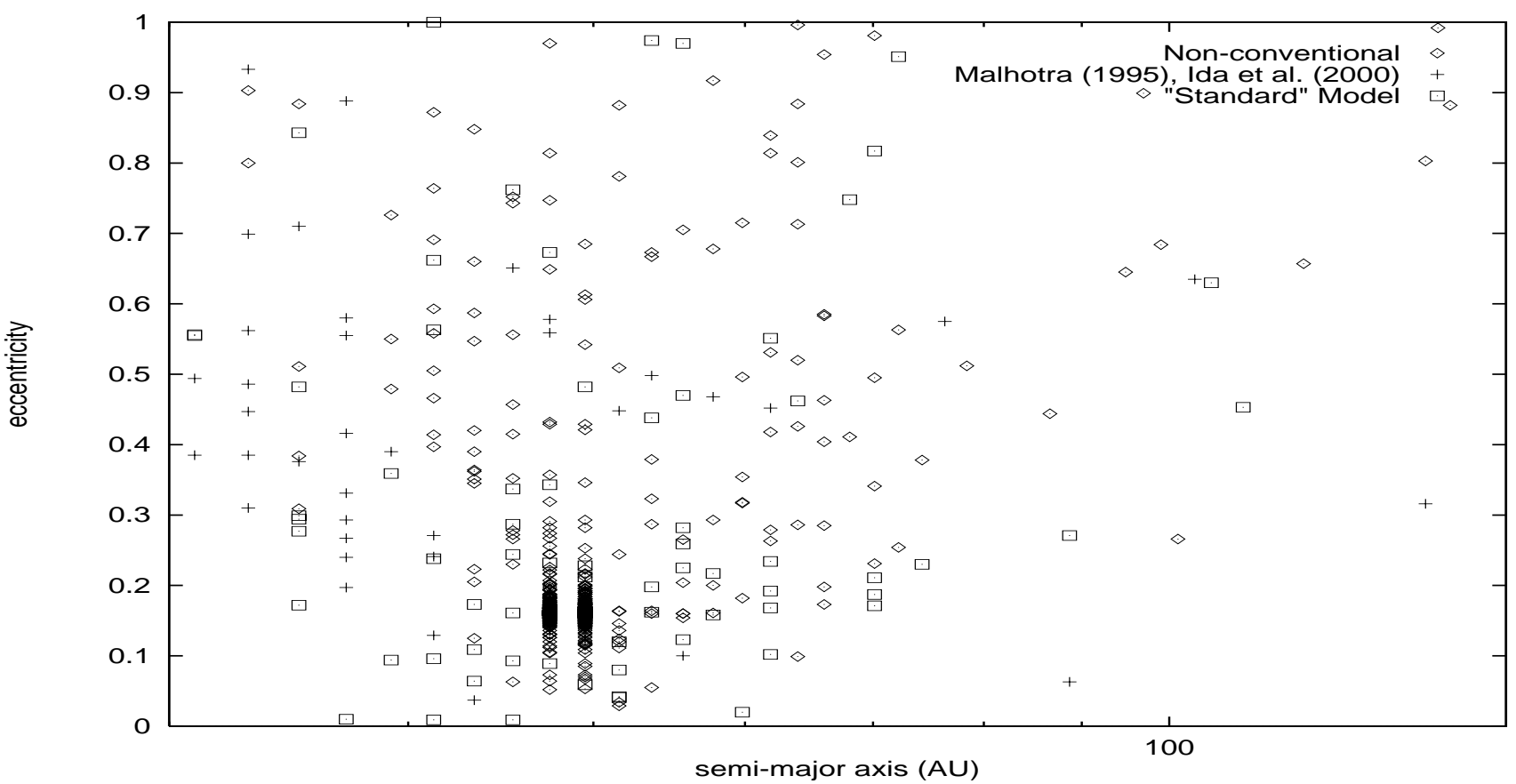

Fig. 2. Cumulative orbital distribution (eccentricity and semi-major axis) of the three distinct original EKB test particles after about 600 Myr. Only systems with changed orbital elements are shown. The results of the three distributions are very different mainly because of the initial eccentricity distributions: almost circular (0.01) orbits for the standard model and $e_{\mathrm{o}}=0.16$ for the non-conventional distribution. The fraction of modified systems approaches $100 \%$ for the non-conventional model and it is less than $20 \%$ for the other two initial conditions. Non-conventional initial conditions produce a large number of slightly modified systems with variations less than a few percent relative to the initial values of the orbital elements. This is mainly because nearly circular or short (39 AU) orbits are very hard to perturb

range $0.01-0.16$ (see Fig. 1). These are our starting values for the primordial EKBO population that we consider in our $N$-body simulations assuming the capture-in-vortex scenario. The numerical values (radial distance and eccentricity) depend strongly on the nebula model used in the calculations. The location of our resonance is for the nebula model described in (e.g.) Cuzzi et al. (1993).

\subsection{N-body calculations}

We have performed a series of $N$-body simulations with NBODY5 (Aarseth 1994) as described in Sect. 2 with three sets of initial conditions. The first set corresponds to the stage before Neptune's outward migration and primordial EKBOs are in almost circular $(e=0.01)$ orbits with semimajor axes distributed almost uniformly in the range 35$60 \mathrm{AU}$ (standard model). The second model assumes the stage after Neptune's outward migration, EKBOs are concentrated at $39 \mathrm{AU}$ with $e \sim 0.1-0.3$ (Malhotra 1995; Ida et al. 2000b), with the high eccentricity being induced by (adiabatic) 3:2 resonant sweeping. The third set considers eccentric (0.16) orbits at $48.5 \mathrm{AU}$ (non-standard model). The results for the three initial conditions are very different and they may help us to revise and improve models of how the outskirts of planetary systems form and evolve.

A comparison among the evolution of the three different scenarios is shown in Fig. 2. Almost circular initial orbits (standard model) are hard to perturb and the fraction of modified systems (including ejected EKBOs) is only $20 \%$ after a time scale of about $600 \mathrm{Myr}$. On the other hand, the 3:2 resonant primordial EKBOs are also hard to disturb with less than $20 \%$ of systems being perturbed and the orbital distribution evidences a lack of wide systems with almost no objects beyond 60 AU. However, a moderately eccentric initial distribution for the non-conventional model produces wider systems and a fraction of modified systems near $100 \%$ although the orbits of most of them are only slightly modified.

The observational distribution of trans-Neptunian and related objects are shown in Figs. $4-7$. The distribution in eccentricity presents a strong peak for almost circular orbits but this could be due to observational errors.

In the non-conventional scenario and following Weidenschilling (1994), we assume that trapped, metresized particles accrete into primordial EKBOs without significant orbital modification in a short time scale after trapping. Subsequent orbital changes could be the result of both multi-planet interaction and stellar encounters in star clusters. In the calculations we restrict ourselves to the latter hypothesis. Our computations show that both an increase and a diminution of orbital eccentricity are achievable although increase is by far the most probable event. The eccentricity variations are associated with gravitational encounters. Gravitational circularization events are related to two-body interactions and the eccentricity decrement is in the range $10-40 \%$. Close encounters with 


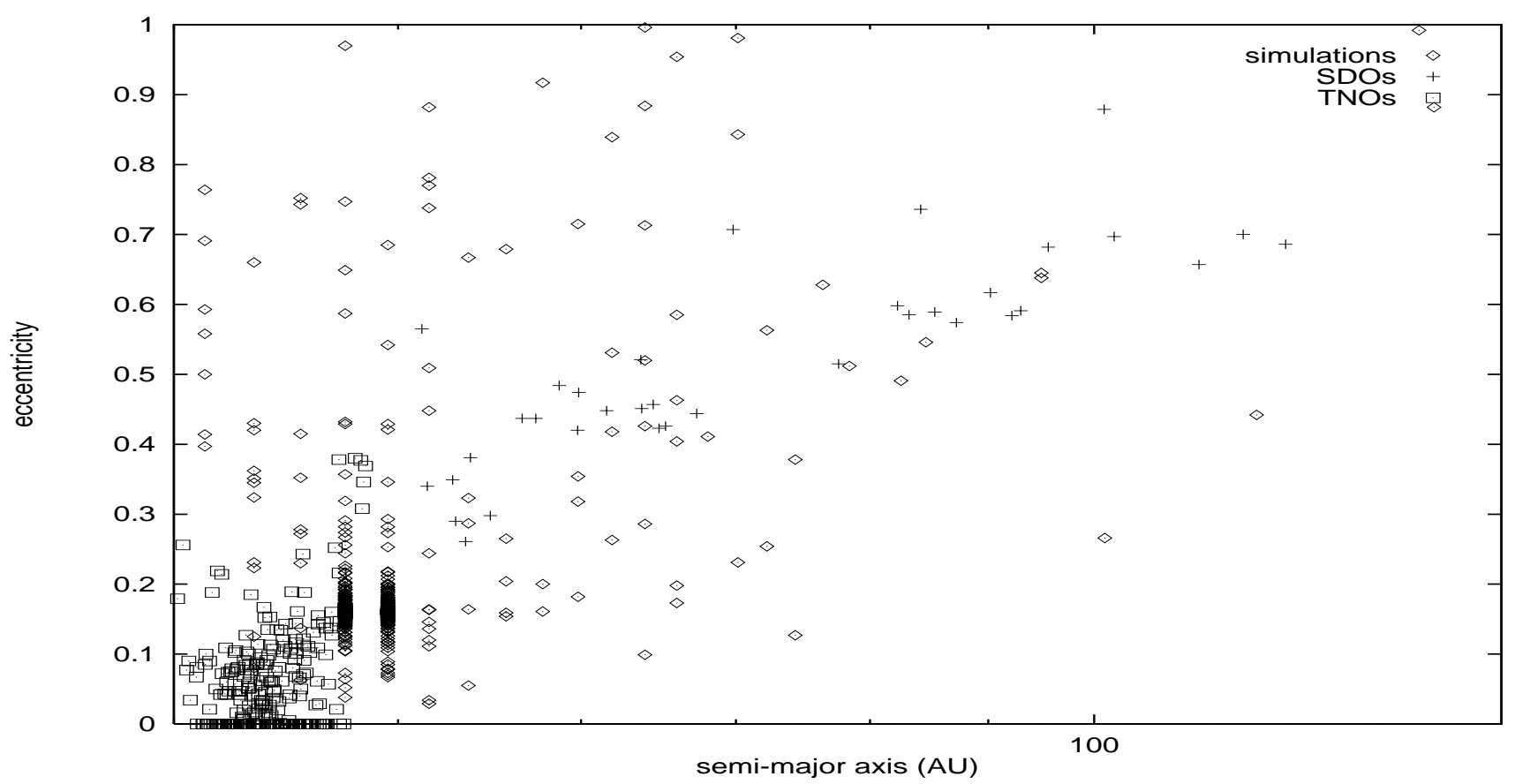

Fig. 3. Orbital distribution (eccentricity and semi-major axis) of the observed trans-Neptunian (TNOs) and scattered disk objects (SDOs) (see, e.g., B. Marsden's Web site, http://cfa-www.harvard.edu/iau/lists/TNOs.html). Most of the objects are located at the 3:2 and 2:1 resonances with Neptune (39.5 and 47.8 AU, respectively). The cumulative orbital distribution of the EKB test particles for the non-conventional model with semi-major axis in the range 40-150 AU (for our 10 models after $500 \mathrm{Myr}$ ) is also shown. The initial eccentricity was about 0.16 and the semi-major axis 48.5 AU. Our orbital distribution does not match very well that of trans-Neptunian objects but it is consistent with the observed orbital distribution of the population of the Scattered Edgeworth-Kuiper Belt (SDOs). Data: 2000 Oct. 27. Although the orbital elements in the observational data are given to several significant figures, many of them are uncertain, and significant changes for individual objects routinely occur as more data are collected. This is particularly severe for objects that have been observed over multiple oppositions

stars in open clusters are able to modify both eccentricities and heliocentric distances of planetesimals leading to scattered EKBOs (see Fig. 3). Our results suggest that the non-standard scenario may be more consistent with the observational distribution of these objects in our solar system.

\subsection{The effects of encounters}

Although three-body scattering plays an essential role in stellar dynamics, the effect of encounters with other stars on the evolution of EKBOs depends on the type of encounter: wide or close. The majority of these interactions are wide, slightly hyperbolic encounters. These quasi-adiabatic interactions are able to change the eccentricity of EKBOs by a small amount (1-10\%) but very effectively if the initial eccentricity is moderately small $(\approx 0.1)$. Larger modifications are associated to temporarily stable configurations (hierarchical systems).

Wide encounters between single stars and systems harboring EKB-like structures result in a change of eccentricity which is generally much larger than the change in semi-major axis of the EKBOs. In this paper an encounter is defined to be wide if the single star passes the EKB system at a distance large enough so as to spread out its disturbing force over several EKBO orbital revolutions; i.e., we assume that the closest distance of approach of the single star, which will be denoted by $r_{\mathrm{p}}$, is still considerably larger than $a$ and the angular velocity of the single star, when it is closest to the EKBO, is considerably smaller than the angular velocity of the EKBOs. In this case the binding energy of the EKBOs is an adiabatic invariant since the effects of the tidal disturbing force nearly cancels over a complete EKBO orbit. Therefore the semimajor axis $a$ of the EKBO, being inversely proportional to the binding energy, is also an adiabatic invariant, and the average change in $a$ falls off exponentially with increasing impact parameter (Heggie 1975). The dynamical situation is not very different if we consider hard binaries instead of single stars as perturbers.

The angular momentum of the EKBO orbit is more sensitive to wide encounters. An intuitive argument can be given by replacing the EKBOs by a massive elliptic ring, with a linear mass density proportional to the time spend by the EKBOs at each part of the ring. The passing star will then exert a torque on this ring, which will transfer angular momentum between the orbit of the single star and the spin of the ring. Replacing the ring by the EKBOs will not change the trajectory of the single star very much because EKBOs are small, and the angular momentum gain/loss of the single star must be balanced by an EKBO orbital angular momentum loss/gain of the 
same magnitude. Since the semi-major axis is hardly affected, the change in angular momentum $J$ of the orbit must be produced mainly by a change in orbital eccentricity $e$, with

$\Delta J^{2} \propto-2 e \Delta e$.

If the torque increases the angular momentum of the EKBO, its eccentricity decreases. An eccentricity increase implies that the angular momentum of the EKBO is decreasing. The intuitive argument given above works only for non-circular orbits but can be extended to also cover circular orbits. By symmetry no torque can be exerted by a passing star on a circular ring, at least not in the plane of the ring (torque components perpendicular to the EKBO orbit do affect the orientation but not the shape of the orbit). However, a more careful argument (Heggie 1975; Rasio \& Heggie 1995) shows that for circular orbits some angular momentum transfer takes place as a higher order effect. Although the orbit is initially circular, it will be deformed periodically by the disturbing force of the passing star. A torque can be exerted on this small deformation by the passing star. It is clear that the torque on the induced asymmetry of the orbit drops off faster for circular orbits than for eccentric orbits which have a permanent asymmetry.

For binaries with non-zero initial eccentricity, $e_{\mathrm{i}}$ it is easy to show that secular perturbation theory (e.g., Danby 1988) gives, in lowest order,

$$
\begin{aligned}
\Delta e= & e_{\mathrm{f}}-e_{\mathrm{i}} \\
= & -\frac{15}{4} \frac{m_{3}}{M_{12} M_{123}}\left(\frac{a}{r_{\mathrm{p}}}\right)^{3 / 2} e \sqrt{1-e^{2}} \mathcal{H}\left(i, e_{\mathrm{p}}, \Omega, \omega\right), \\
\mathcal{H}\left(i, e_{\mathrm{p}}, \Omega, \omega\right)=\frac{1}{\left(1+e_{\mathrm{p}}\right)^{3 / 2}} & \left(\sin ^{2} i \sin 2 \Omega\left(\arccos \left(-1 / e_{\mathrm{p}}\right)+\sqrt{e_{\mathrm{p}}^{2}-1}\right)+\frac{1}{3}\right. \\
& \left(\left(1+\cos ^{2} i\right) \cos 2 \omega \sin 2 \Omega+2 \cos i \sin 2 \omega \cos 2 \Omega\right) \\
& \left.\frac{\left(e_{p}^{2}-1\right)^{3 / 2}}{e_{p}^{2}}\right)
\end{aligned}
$$

(cf. Heggie \& Rasio 1996, Eq. (7)). Here $m_{1}$ and $m_{2}$ are the masses of the binary components (the EKBO system), $m_{3}$ is the mass of the passing object (single star or binary), $M_{12}=m_{1}+m_{2}, M_{123}=M_{12}+m_{3}, a$ is the semi-major axis of the binary (EKBO), and $r_{\mathrm{p}}$ is the closest distance of approach of the third body. The line of nodes is defined to be the line of intersection of the orbital planes of the third body and the binary. Then the ascending node is the part of this line at which the third body crosses the plane of the binary in the direction of the angular momentum vector of the binary. The longitude of the ascending node, $\Omega$, is measured in the plane of motion of the binary (in the sense of the motion of its components) from the direction of pericentre of the binary, $i$ is the inclination of the two orbital planes, and $\omega$ is the longitude of pericentre of the third body, measured in its plane of motion from the ascending node, in the sense of its motion around the binary. The orbit of the third body relative to the barycentre of the binary is hyperbolic with eccentricity $e_{\mathrm{p}}$. For this process, the perturbation reaches its maximum when $e \approx 0.7$ and it declines for very eccentric systems. The effect of this process on the eccentricity of EKBOs depends strongly of the initial value of the eccentricity and therefore on the formation model assumed. Applying Eq. (2), the eccentricity perturbation on an eccentric EKBO orbit (0.16) is about 16 times the value of the perturbation on a classical $(e=0.01)$ orbit. On the other hand, it also depends slightly on the fraction of PBs because the perturbation from a single passing star is about 0.6 times the perturbation from a binary.

Although our present calculations are not intended to analyze systematically the distribution of parameters of the stellar encounters that the planetary systems experience before disintegration of the parent cluster we make some brief remarks here. The dynamical effect of a hard binary population produces two different distributions in eccentricity. Close encounters, without hierarchical system formation, lead to relatively small modifications in the primordial eccentricity but strong interactions inside hierarchical configurations produce high eccentricities. Eccentricity variations of the order of $20 \%$ are by far the most common, 70\%. Our current calculations suggest that close encounters with $\Delta e>0$ are more common. Variations within $20 \%$ are produced for $r_{\mathrm{p}} / a$ in the range 8-13. As regards the semi-major axis, it may increase as a result not only of external perturbations but also because of mass loss from the parent star.

\section{Discussion}

In general, the small bodies populating the outer solar system are named EKBOs, however the orbits of these bodies are not randomly distributed within the Belt but can be grouped into three classes. The classical EKBOs constitute about two-thirds of the currently known objects and they are characterized by relatively small eccentricities (around 0.1) and semi-major axes in the range 41-46 AU. The second group, the resonant EKBOs, are trapped in mean-motion resonances with Neptune, mainly the 3:2 resonance at $39.4 \mathrm{AU}$. The third dynamical class, discovered in 1996 (Duncan \& Levison 1997), is characterized by large eccentricities and perihelia in the range 34$36 \mathrm{AU}$ and its members are called Scattered EdgeworthKuiper Belt objects (SEKBOs) or Scattered Disk Objects (SDOs). Our calculations suggest that this third class may be the result of close and distant encounters between the proto-Sun and other members of its parent star cluster. This result is consistent with the conclusions of Ida et al. (2000a) although our experimental approaches are rather different. However, Ida et al. (2000a) does not explicitly propose that the SD was formed by a stellar encounter but the $e \sim 0.1$ in the classical EKB has been pumped up by a stellar encounter. The orbital distribution of these 


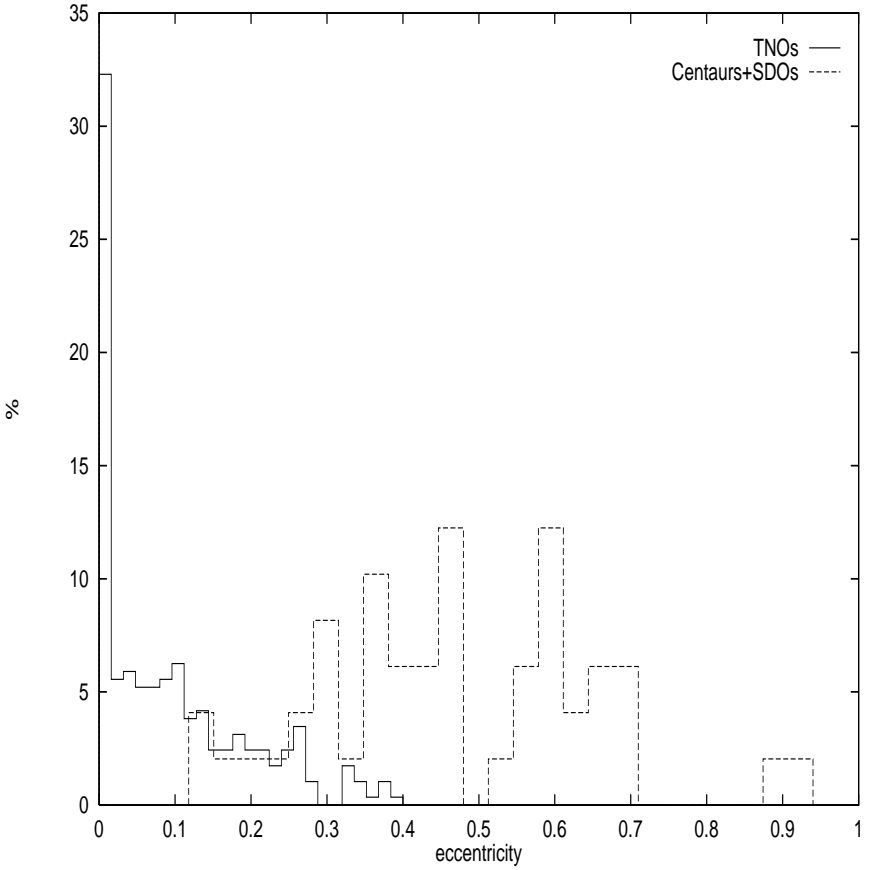

Fig. 4. Eccentricity distribution in the TNO sample (solid line) and Centaur+SDO sample (dashed line). The TNOs have eccentricities in the range $[0,0.4]$ but the Centaur+SDOs sample's eccentricities are mainly in the range [0.1, 0.7]. Data source as in Fig. 3

objects has remained relatively unperturbed since the escape of the proto-Sun from its parent cluster. Our results suggest that if the present distribution of the orbital elements of the solar system bodies currently classified as scattered disk objects is the result of multi-body gravitational interactions of the solar protoplanetary system with other stars in its parent star cluster, the Sun remained as bound member for at least 500 Myrs. The evolution of a short-lived, $\approx 200$ member cluster is unable to produce significant modification of the primordial distribution of the orbital elements of EKBOs. It means that the solar parent cluster may have an original population of about 500-800 members (a real open cluster not an association) or twice this number if we consider individual components of binary stars. Adams \& Laughlin (2001) have found a similar result using Monte-Carlo simulations with a planet scattering code (Laughlin \& Adams 1998, 2000). Figures 4 and 6 show the observational eccentricity distribution of the EKBO sample in our own solar system. This distribution exhibits a lack of objects with eccentricity higher than 0.7 which is fully consistent with Eq. (2): the perturbing efficiency of distant encounters decreases for systems with eccentricity higher than 0.7. However, this observational evidence is not fully conclusive since most large eccentricity orbits are removed through close encounters with Neptune, truncating the eccentricity distribution. The situation seems to be very different for the EKB-like structure around $\epsilon$ Eridani (Greaves et al. 1998). The $\epsilon$ Eridani ring system appears close to face-on with roughly circular morphology (the major/minor axis ratio is $\approx 1.1$ ), with

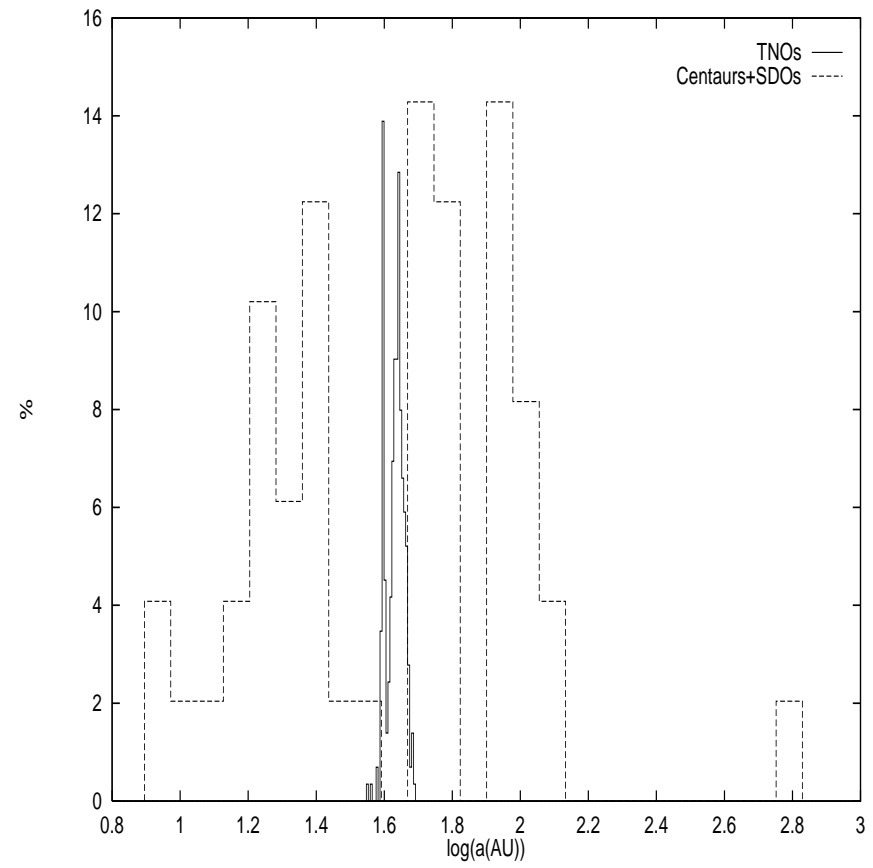

Fig. 5. $\log (a)$ distribution in the TNO sample (solid line) and Centaur+SDO sample (dashed line). Data source as in Fig. 3. Most of the TNOs are located at the $3: 2$ and 2:1 resonances with Neptune (39.5 and 47.8, respectively)

the dust ring aligned with the equator of the host star. $\epsilon$ Eridani is a main-sequence star of type $\mathrm{K} 2 \mathrm{~V}\left(0.8 M_{\odot}\right)$ with an estimated age of 0.5-1.0 Gyr, hence if the observed structure is almost circular this star could be born in a short-lived $(<0.1 \mathrm{Gyr})$, small cluster $(<100$ members $)$ otherwise, the morphology of the ring system should be more eccentric. Early escape from a larger cluster is also compatible with the observational results however there are no young clusters close enough. Preferential escape of low-mass stars is a well known feature of the dynamical evolution of star clusters. Evidence for a long-period, eccentric planet around $\epsilon$ Eridani has been recently provided (Hatzes et al. 2000)

Our results and discussion are not meant to provide a unique or complete model of either the formation of the EKB in our solar system or similar structures observed around other stars. Instead we simply point out the dynamical signatures expected depending on the environmental conditions where new-born stars and structures around them evolve.

Acknowledgements. We thank Dr. S. J. Aarseth for providing his computer code. CFM is very grateful to his Ph.D. Thesis supervisor Dr. Pierre Barge for his useful comments on the vortex model of planet formation. The authors thank the Department of Astrophysics of Universidad Complutense de Madrid for allotting excellent computing facilities. Part of the calculations were performed on the SGI Origin 2000 of the "Centro de Supercomputación Complutense". We would like to thank the referee, S. Ida. His suggestions have lead to a substantially improved paper. In preparation of this paper, we made use of the Minor Planet Center database at CfA, Harvard, USA, the ASTRO-PH e-print server, the NASA Astrophysics 


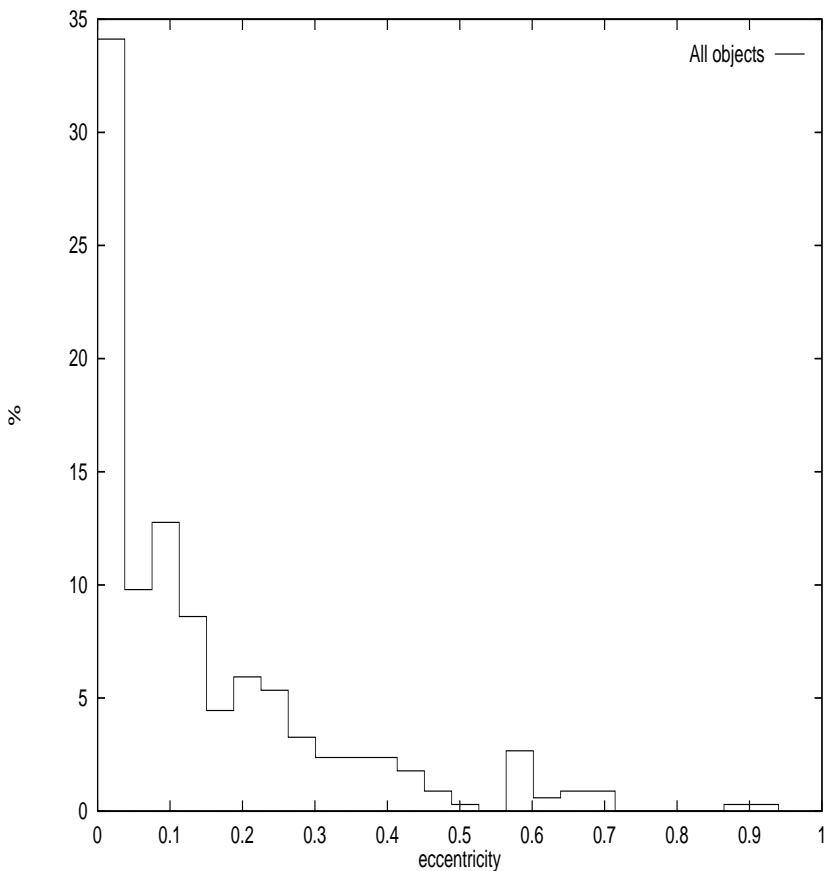

Fig. 6. Eccentricity distribution in the observational sample including all the objects in Fig. 4. Data source as in Fig. 3

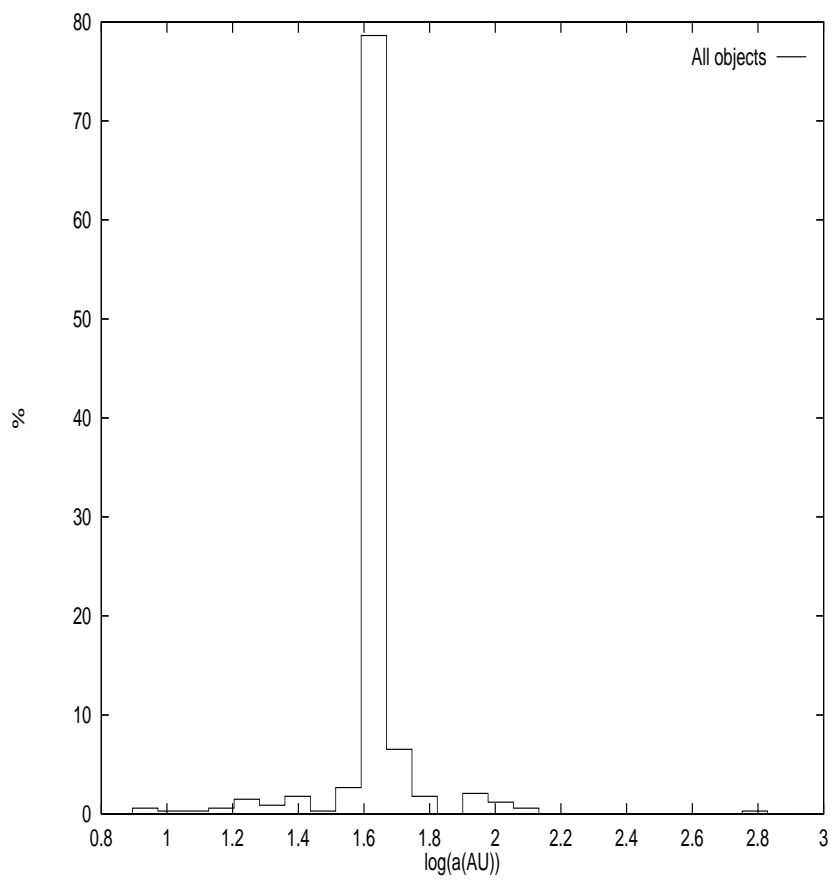

Fig. 7. $\log (a)$ distribution in the observational sample including all the objects in Fig. 5. Data source as in Fig. 3

Data System, and the WEBDA database operated at the IoA, University of Lausanne, Switzerland.

\section{References}

Aarseth, S. J. 1994, in Galactic Dynamics and $N$-body Simulations, ed. G. Contopoulos, N. K. Spyrou, \& L. Vlahos (Springer Verlag, Berlin), 277

Aarseth, S. J., Hénon, M., \& Wielen, R. 1974, A\&A, 37, 183

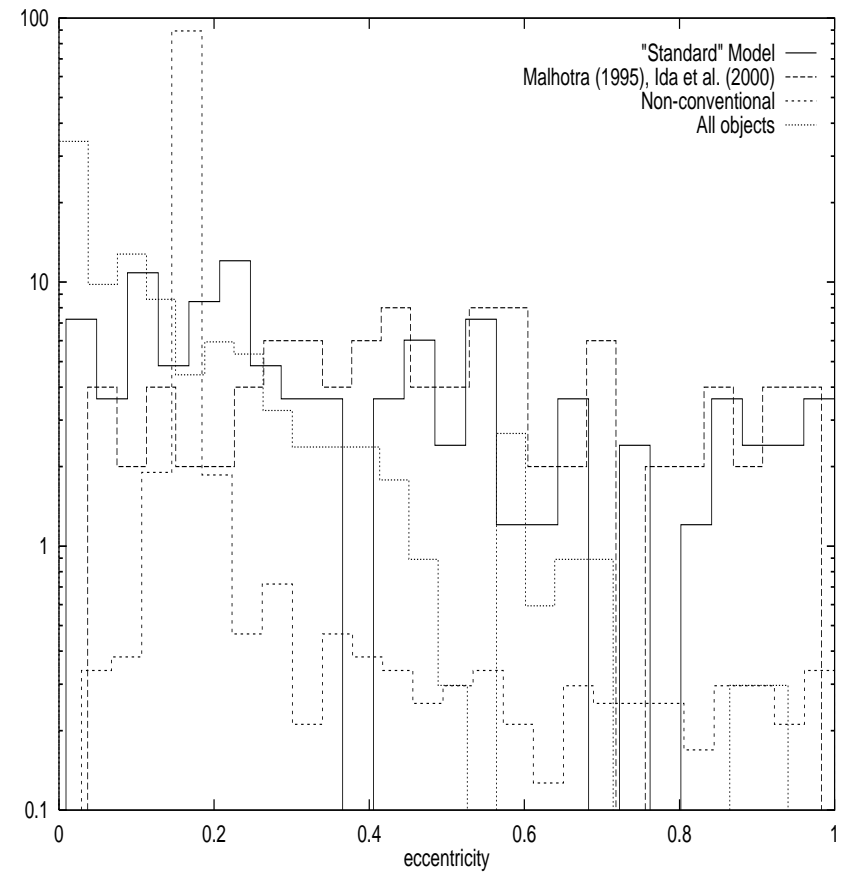

Fig. 8. Eccentricity distribution in the observational sample and the three EKBO test populations

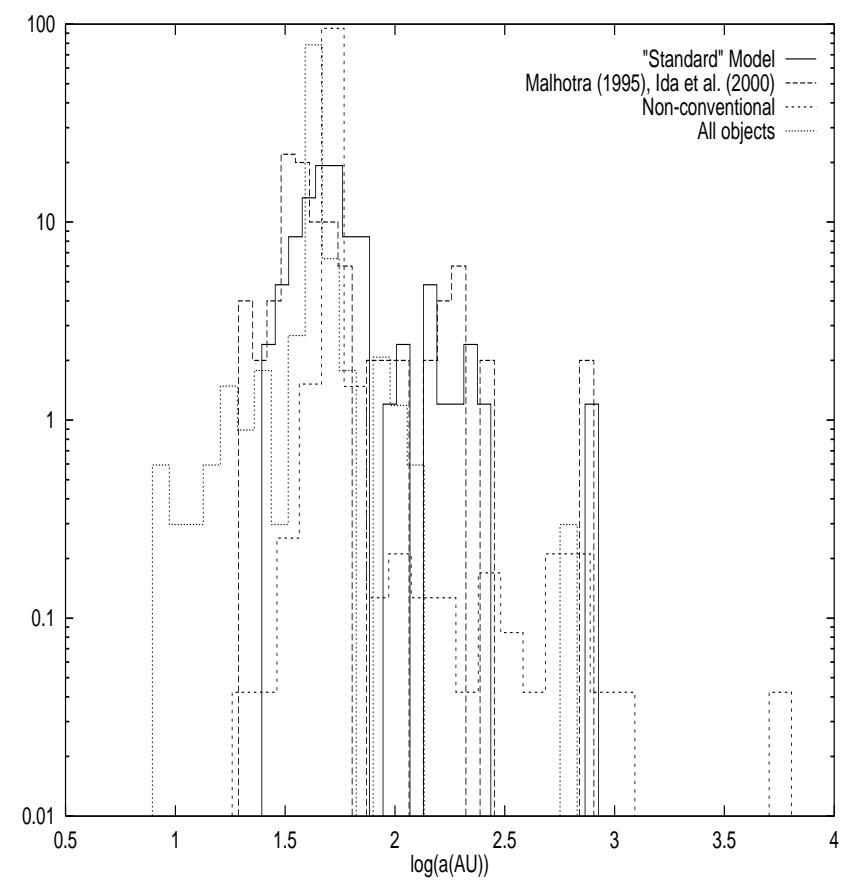

Fig. 9. $\log (a)$ distribution in the observational sample and the three EKBO test populations

Adams, F. C., \& Laughlin, G. 2001, Icarus, 150, 151

Barge, P., \& Sommeria, J. 1995, A\&A, 295, L1

Beckwith, S. V. W., Henning, T., \& Nakagawa, Y. 2000, in Protostars \& Planets IV, ed. V. Mannings, A. P. Boss, \& S. S. Russell (University of Arizona Press, Tucson), 533

Boss, A. P. 1988, Comm. Astrophys., 12, 169

Bracco, A., Provenzale, A., Spiegel, E. A., \& Yecko, P. 1998, in Quasars and Accretion Disks, ed. A. Abramowicz (Cambridge University Press, Cambridge), 254 
Bracco, A., Chavanis, P.-H., Provenzale, A., \& Spiegel, E. A. 1999, Physics of Fluids, 11, 2280

Bryden, G., Lin, D. N. C., \& Ida, S. 2000, ApJ, 544, 481

Bulirsch, R., \& Stoer, J. 1966, Numer. Math., 8, 1

Chavanis, P-H. 2000, A\&A, 356, 1089

Cuzzi, J. N., Dobrovolskis, A. R., \& Champney, J. M. 1993, Icarus, 106, 102

Danby, J. M. A. 1988, Fundamentals of Celestial Mechanics, Willmann-Bell, Richmond

Dominik, C., Laureijs, R. J., Jourdain de Muizon, M., \& Habing, H. J. 1998, A\&A, 329, L53

Duncan, M., \& Levison, H. 1997, Science, 276, 1670

Eggleton, P. P., Fitchett, M. J., \& Tout, C. A. 1989, ApJ, 347, 998

de la Fuente Marcos, R. 1997, Ph.D. Thesis, Universidad Complutense de Madrid

de la Fuente Marcos, C., \& Barge, P. 2001, MNRAS, 323, 601

de la Fuente Marcos, C., \& de la Fuente Marcos, R. 1997, A\&A, $326, \mathrm{~L} 21$

de la Fuente Marcos, C., \& de la Fuente Marcos, R. 1998a, NewA, 4, 21

de la Fuente Marcos, C., \& de la Fuente Marcos, R. 1998b, EM\&P, 81, 145

de la Fuente Marcos, R., \& de la Fuente Marcos, C. 2000a, in Stellar Clusters and Associations: Convection, Rotation, and Dynamos, ed. R. Pallavicini, G. Micela, \& S. Sciortino, ASP Conf. Ser., San Francisco, 198, 183

de la Fuente Marcos, R., \& de la Fuente Marcos, C. 2000b, Ap\&SS, 271, 127

de la Fuente Marcos, C., \& de la Fuente Marcos, R. 2001, in Modes of Star Formation and the Origin of Field Populations, ed. E. Grebel, \& W. Brandner, ASP Conf. Ser., San Francisco, in press

Godon, P., \& Livio, M. 1999a, ApJ, 521, 319

Godon, P., \& Livio, M. 1999b, ApJ, 523, 350

Godon, P., \& Livio, M. 2000, ApJ, 537, 396

Goldreich, P., \& Ward, W. R. 1973, ApJ, 183, 1051

Greaves, J. S., Holland W. S., Moriarty-Shieven, G., et al. 1998, ApJ, 506, L133

Grinin, V., Natta, A., \& Tambovtseva, L. 1996, A\&A, 313, 857

Hatzes, A. P., Cochran, W. D., McArthur B., et al. 2000, ApJ, 544, L145

Heggie, D. C. 1975, MNRAS, 173, 729

Heggie, D. C., \& Rasio, F. A. 1996, MNRAS, 282, 1064

Ida, S., Larwood, J., \& Burkert, A. 2000a, ApJ, 528, 351

Ida, S., Bryden, G., Lin, D. N. C., \& Tanaka, H. 2000b, ApJ, 534,428
Ingersoll, A. P. 1990, Science, 248, 308

Jewitt, D. C, \& Luu, J. X. 2000, in Protostars \& Planets IV, ed. V. Mannings, A. P. Boss, \& S. S. Russell (University of Arizona Press, Tucson), 1201

Kenyon, S. J., \& Luu, J. X. 1998, AJ, 115, 2136

Laughlin, G., \& Adams, F. C. 1998, ApJ, 508, L171

Laughlin, G., \& Adams, F. C. 2000, Icarus, 145, 614

Lin, D. N. C. 1981, ApJ, 246, 972

Malhotra, R. 1995, AJ, 110, 420

Marsden, B. 2000, http://cfa-www.harvard.edu/iau/lists/centaurs.html http://cfa-www.harvard.edu/iau/lists/TNOs.html

Mermilliod, J.-C. 2000, The database for stars in open clusters, http://obswww . unige.ch/webda

Miller, J., Weichman, P. B., \& Cross, M. C. 1992, Phys. Rev. A, 45,2328

Morfill, G. E. 1985, in Birth and Infancy of Stars (North Holland Press, Amsterdam), 693

Nezlin, M. V., \& Snezhkin, E. N. 1993, Rossby Vortices, Spiral Structures, Solitons (Springer-Verlag, Berlin)

Palme, H., \& Boynton, W. V. 1993, in Protostars \& Planets III, ed. E. H. Levy, J. L. Lunine, \& M. S. Matthews (University of Arizona Press, Tucson), 979

Rasio, F. A., \& Heggie, D. C. 1995, ApJ, 445, L133

Safronov, V. S. 1969, Evolution of the Protoplanetary Cloud and Formation of the Earth and the planets, Nauka, Moscow (in Russian, English translation in NASA TTF$667)$

Scholl, H., Roques, F., \& Sicardy, B. 1993, Cel. Mech. \& Dyn. Astron., 56, 381

Schmitt, W., Henning, T., \& Mucha, R. 1997, A\&A, 325, 569

Sommeria, J., Meyers, S. D., \& Swinney, H. L. 1988, Nature, 331,689

Sommeria, J., Nore, C., Dumont, T., \& Robert, R. 1991, C. R. Acad. Sci. Paris II, 312, 999

Stern, S. A., \& Colwell, J. E. 1997, AJ, 114, 841

Tanga, P., Babiano, A., Dubrulle, B., \& Provenzale, A. 1996 Icarus, 121, 158

Trilling, D. E., Brown, R. H., \& Rivkin, A. S. 2000, ApJ, 529, 499

Weidenschilling, S. J. 1980, Icarus, 44, 172

Weidenschilling, S. J. 1994, Nature, 368, 721

Weidenschilling, S. J. 1995, Icarus, 116, 433

Weidenschilling, S. J. 1997, Icarus, 127, 290 